\title{
Correction to: Content Based Routing Algorithm to Improve QoS in IoMT Networks
}

Shailja Agnihotri and K. R. Ramkumar

Correction to:

Chapter "Content Based Routing Algorithm to Improve QoS in IoMT Networks" in: A. Saha et al. (Eds.): Advances

in Computational Intelligence, Security and Internet of Things, CCIS 1192, https://doi.org/10.1007/978-981-15-3666-3_17

In the originally published version of paper starting on p. 195, the affiliation of the second author K. R. Ramkumar was incomplete. The affiliation has been extended as "Chitkara University Institute of Engineering and Technology, Chitkara University, Punjab, India". 